\title{
Shock-frequency reduction and avoidance behavior in a running wheel*
}

\author{
CHARLES R. CROWELL \\ McMaster University, Hamilton, Ontario, Canada L8S $4 k 1$
}

\begin{abstract}
The effect of response-contingent shock-frequency reduction upon the strength of a locomotor avoidance response was studied in a three-phase procedure. In the first and third phases, a locomotor response resulted in a reduction in the probability of shock per 2-sec interval from 0.5 to 0.1 . In the second phase, however, the probability of shock was fixed at 0.5 , regardless of locomotor behavior. It was observed that Ss started Phase 1 with a relatively strong tendency to perform the locomotor response that was maintained throughout that phase. In the second phase, locomotor behavior declined to a near-zero level while substantial recovery of the response tendency was noted in the final phase. Whereas these results are consistent, in some respects, with the supposition the shock-frequency reduction can serve as a source of negative reinforcement, the failure to observe an initial increase in performance during Phase 1 suggests an alternative interpretation of the present results.
\end{abstract}

Herrnstein and Hineline (1966) proposed and tested a novel avoidance-conditioning procedure. In their experiment, shocks were programmed to occur at 2 -sec "step" intervals with a certain probability. The probability of shock per step at any given time was determined by whether a response or a shock had occurred most recently. Typically, although not always, a higher probability of shock was delivered if a shock had occured last (postshock schedule). Following a response, the probability of shock was reduced (postresponse schedule) until the next programmed shock occured at which time the probability in effect reverted to the higher value again. On the average, this schedule provided a reduction in the frequency of shock following a response that was proportional to the difference in the two probability values. If the two probabilities were equal then no reduction in the frequency of shock occurred, on the average.

In their experiment, Herrnstein and Hineline explored the effects of several pairs of probability values and noted that the strength of a barpress response in rats was related to the amount of shock-frequency reduction following that response. This "random shock" procedure was thought, by the authors, to have eliminated all obvious candidates for an explicit conditioned stimulus (CS), and thus the apparent learning under this procedure was assumed to indicate that such a CS was not a prerequisite for avoidance conditioning.

Rossi and Rilling (1971) have confirmed the findings of Herrnstein and Hineline (1966) in an investigation employing the treadle response in pigeons as a dependent measure. The purpose of the present study

\footnotetext{
*This research was completed while the author was at the University of Iowa. Support for this project was provided by National Institute of Mental Health Research Grants MH 11734-04 and MH 23607-01 to Judson S. Brown. The author wishes to thank Dr. Brown, who is the sponsor of this article, and also John R. Platt for their assistance in the preparation of this manuscript. Requests for reprints should be sent to the author at the Department of Psychology, McMaster University, Hamilton, Ontario, Canada.
}

was to extend the generality of the above findings to locomotor avoidance behavior in a running wheel.

\section{METHOD}

\section{Subjects}

The Ss employed in this study were five naive female albino rats, 70 days old at the start of the experiment. They were obtained from Carworth Farms, Inc., Portage, Michigan (Sprague-Dawley derivations), and were individually housed and maintained under constant-light conditions with free access to food and water.

\section{Apparatus}

The apparatus was a small rodent running wheel (Lehigh Valley Electronics, Model 147-11) $23 \mathrm{~cm}$ in diam and $7 \mathrm{~cm}$ wide, modified so that wheel movement was unidirectional. The running surface of the wheel was constructed of $1.5-\mathrm{mm}$ in diam stainless steel rods spaced $0.64 \mathrm{~cm}$ apart center to center. Every other grid in the wheel was wired in common and a commutator allowed the grids to be electrified. A response was defined as a quarter turn of the wheel on the average. Electric shock was produced by a Grason-Stadler shock generator (Model E1064GS) set for a nominal intensity value of $0.3 \mathrm{~mA}$. All shocks were $0.3 \mathrm{sec}$ in duration.

\section{Procedure}

This experiment consisted of three phases: an initial acquisition phase in which the postshock and postresponse probabilities ere 0.5 and 0.1 , respectively; an extinction phase during whic. both probabilities were 0.5 ; and a reacquisition phase similar to the initial phase. Each of the five Ss was administered the same sequence of phases. The probabilities were determined by two schedules of irregularly spaced holes punched in a multichannel paper tape. This tape was advanced at the rate of one step every $2 \mathrm{sec}$ throughout each of the 20 -min experimental sessions. One channel of the tape determined the postshock probability, while the other channel controlled the postresponse probability.

The occurrence of a hole in the effective channel resulted in the delivery of a brief shock to a rat in the running wheel. With a 0.5 probability of shock in effect, a hole occurred, on the average, every second tape step which resulted in an average intershock interval of $4 \mathrm{sec}$. A 0.1 probability schedule, however, produced an average intershock interval on the tape of $20 \mathrm{sec}$, since only one shock would occur out of 10 steps. Each session 


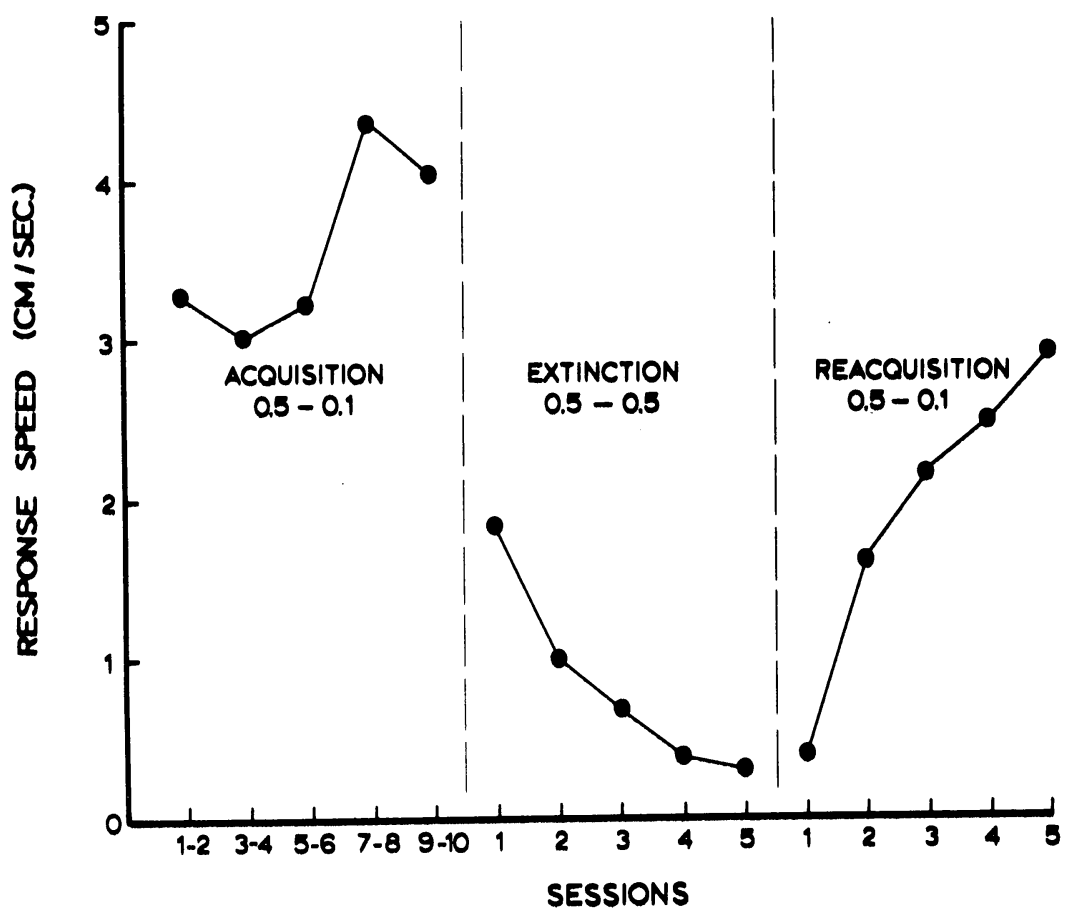

Fig. 1. Mean locomotor response speed as a function of sessions during acquisition, extinction, and reacquisition phases.

was started with the postshock schedule in effect and a locomotor response resulted in a shift of control of shock delivery from the postshock to the postresponse schedule. Control of shock delivery remained with the postresponse schedule until the next shock was delivered, whereupon control was automatically transferred back to the postshock schedule. Locomotor behavior during the postresponse schedule had no programmed consequences. A total of 20 sessions was administered, 10 of acquisition and 5 each of extinction and reacquisition.

\section{RESULTS}

The mean response latency from the onset of the postshock schedule was computed for every rat during each session and was transformed into a response speed in $\mathrm{cm} / \mathrm{sec}$. The group speed function over sessions is plotted in Fig. 1 for each of the three phases. During the initial acquisition phase, sessions were blocked by twos.

Figure 1 indicates that the Ss tended to start training with relatively high levels of performance. The apparent increase in speed over the course of acquisition was not significant. During the first session of extinction, response speed declined resulting in a mean speed on that day that was substantially slower than on the last day of acquisition. A progressive and significant decline in response speed was observed over the subsequent four sessions of extinction $[F(4,16)=5.49, p<.05]$. A consistent and significant increase in speed was noted over the course of reacquisition $[\mathrm{F}(4,16)=11.99$, $\mathrm{p}<.05]$.

An alternative measure of performance in this situation is based upon the number of programmed shocks that are "avoided" by a $\mathrm{S}$ in a session. This measure is analogous to conventional measures of percent avoidance and may be calculated as follows:

$$
\text { percent avoidance }=\frac{\mathrm{Max}-\mathrm{Obt}}{\mathrm{Max}-\mathrm{Min}} \times 100 .
$$

where Max refers to the maximum number of shocks that would be delivered (on the average) in the absence of responding; $\mathrm{Obt}$ refers to the number of shocks obtained by a S; and Min refers to the minimum number of shocks that a $\mathrm{S}$ could receive in a session (i.e., the number programmed to occur on the postresponse schedule alone).

Figure 2 depicts the percent avoidance function during acquisition and reacquisition. The function based on this measure during extinction is not informative since, by definition, no shocks can be avoided in that phase. It may be noted that these functions are comparable with those based upon the speed measure. During acquisition, the change in performance from the initial block to the final block is not significant. In the reacquisition phase, however, a significant increase in percent avoidance was noted over sessions $[F(4,16)=$ $20.46, p<.05]$.

\section{DISCUSSION}

The results of this study are similar to those of Herrnstein and Hineline (1966) and Rossi and Rilling (1971) in that a response leading to a reduction in the frequency of aversive stimulation was observed to increase in strength. Unlike the previous studies, however, this increase in response strength was not observed 
Fig. 2. Mean percent avoidance as a function of sessions during acquisition and reacquisition phases.

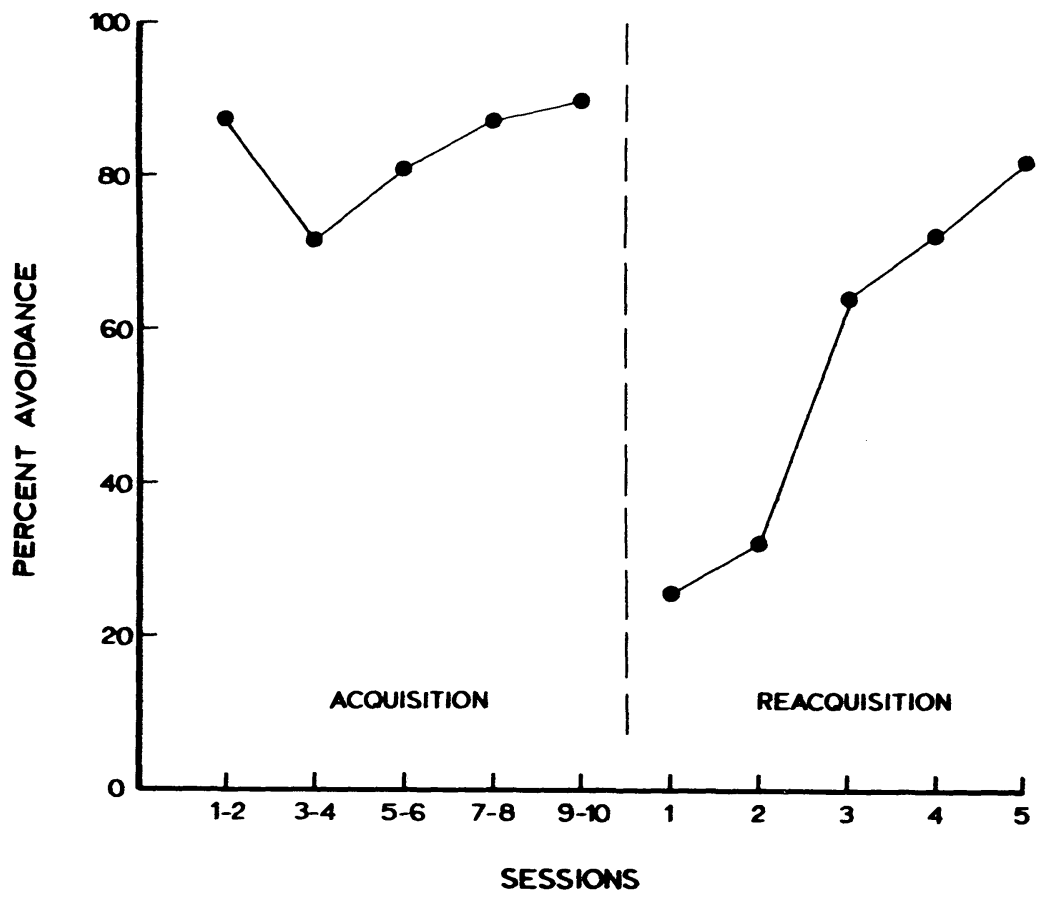

during the initial acquisition phase. Moreover, in sharp contrast to the findings of Herrnstein and Hineline (1966), the changes in locomotor behavior observed in this study during extinction and reacquisition were relatively rapid, occurring within a few sessions. These results may be interpreted as further support for the conjecture that reductions in shock rate following responses are a source of negative reinforcement if the failure to obtain an increase in performance during the first acquisition phase is attributed to some factor such as the tendency for shocks to elicit locomotor responses early in training. Since this tendency might be expected to decline in strength as a function of the organism's repeated experience with shock, the maintenance of locomotor behavior in the latter stages of the first acquisition period could have been due, in part, to the reinforcing effects of response-contingent shock-rate reduction. During extinction, however, responses were not followed by a reduction in the frequency of shock and were of ten followed by an immediate shock. Thus, the decline in the tendency to respond noted during this phase may have resulted from some combination of extinction and punishment processes. On this view, the behavior observed during reacquisition seems to reflect most clearly the presumed reinforcing effects of response-contingent shock-rate reduction since progressive increases in percent avoidance and response speed were noted.

In the face of a lack of improvement in performance during initial acquisition, however, an alternative interpretation of the results of this study can be formulated that involves only punishment rather than negative reinforcement processes. According to this view, the maintenance of performance during the first acquisition period would be attributed solely to whatever factors were responsible initially for the appearance of the locomotor behavior (e.g., shock elicited running). The decline in locomotor behavior during extinction would be regarded as an example of the punishing effects of close temporal proximity of responses and shock provided by the extinction procedure. The recovery of locomotor behavior during reacquisition would represent "extinction" of the effects of the prior punishment and the consequent reinstatement of the factors responsible for locomotion in the first phase.

Thus, the results of this study do not provide unambiguous support for the supposition that locomotor behavior is strengthened by response-contingent reductions in the frequency of shock, a notion implicit in the agruments advanced by Herrnstein (1969). Manipulations such as those involving the absolute frequency of shocks delivered by the postshock schedule should allow the alternative interpretations of the results of this study to be unraveled, inasmuch as this factor could influence the extent or magnitude of shock elicited running.

\section{REFERENCES}

Herrnstein, R. J. Method and theory in the study of avoidance. Psychological Review, 1969, 76, 49-69.

Herrnstein, R. J., \& Hineline, P. N. Negative reinforcement as shock-frequency reduction. Journal of the Experimental Analysis of Behavior, 1966, 9, 421-430.

Rossi, R., \& Rilling, M. Effects of shock frequency reduction on avoidance responding in the pigeon. Psychonomic Science, 1971, 25, 267-268.

(Received for publication March 1, 1974.) 